Popovych Daria V., Badiuk Nataliya S., Hrytsak Myroslava V., Ruzhylo Sofiya V., Mel'nyk Oksana I., Żukow Xawery. Sulfatechloride sodium-magnesium mineral waters modulate neuroendocrine-immune complex and metabolism in healthy female rats. $\begin{array}{lllllll}\text { Journal of Education, Health and } & \text { Sport. } & \text { 2021;11(12):455-466. } & \text { eISSN }\end{array}$ http://dx.doi.org/10.12775/JEHS.2021.11.12.037

https://apcz.umk.pl/czasopisma/index.php/JEHS/article/view/JEHS.2021.11.12.037

https://zenodo.org/record/6320628

The journal has had 40 points in Ministry of Education and Science of Poland parametric evaluation. Annex to the announcement of the Minister of Education and Science of December 21, 2021. No. 32343. Has a Journal's Unique Identifier: 201159. Scientific disciplines assigned: Physical Culture Sciences (Field of Medical sciences and health sciences); Health Sciences (Field of Medical Sciences and Health Sciences).

Punkty Ministerialne z 2019 - aktualny rok 40 punktów. Zalącznik do komunikatu Ministra Edukacji i Nauki z dnia 21 grudnia 2021 r. Lp. 32343 . Posiada Unikatowy Identyfikator Czasopisma: 201159. Przypisane dyscypliny naukowe: Nauki o kulturze fizycznej (Dziedzina nauk medycznych i nauk o zdrowiu); Nauki o zdrowiu (Dziedzina nauk medycznych i nauk o zdrowiu).

(1) The Authors 2021;

This article is published with open access at Licensee Open Journal Systems of Nicolaus Copernicus University in Torun, Poland Open Access. This article is distributed under the terms of the Creative Commons Attrioution Noncommercial License which permits any noncommercial use, distribution, and reproduction in any medium, provided the original author (s) and source are credited. This is an open access articte and reproduction in any medium, provided the work is properly cited. The authors declare that there is no conflict of interests regarding the publication of this paper.

Received: 01.12.2021. Revised: 20.12.2021. Accepted: 31.12.2021.

\title{
SULFATE-CHLORIDE SODIUM-MAGNESIUM MINERAL WATERS MODULATE NEUROENDOCRINE-IMMUNE COMPLEX AND METABOLISM IN HEALTHY FEMALE RATS
}

\author{
Daria V. Popovych ${ }^{1}$, Nataliya S. Badiuk ${ }^{1}$, Myroslava V. Hrytsak ${ }^{2,3}$, \\ Sofiya V. Ruzhylo ${ }^{4}$, Oksana I. Mel'nyk $^{5}$, Xawery Żukow ${ }^{6}$ \\ ${ }^{1}$ IY Horbachevs'kyi National Medical University, Ternopil', Ukraine \\ darakoz@yahoo.com \\ ${ }^{2}$ SE Ukrainian Research Institute of Medicine of Transport, Odesa, Ukraine \\ badiuknns@ukr.net \\ ${ }^{3}$ Scientific group of Balneology of Hotel\&Spa Complex "Karpaty", Truskavets', \\ Ukraine hrytsak.myroslava@gmail.com ira barschyk@ukr.net \\ ${ }^{4}$ Ivan Franko Pedagogical University, Drohobych, Ukraine \\ 5'Danylo Halyts'ky̌̌ National Medical University, L'viv, Ukraine omelnyk7@gmail.com \\ ${ }^{6}$ Medical University of Bialystok, Bialystok, Poland xaweryzukow@gmail.com
}

Background. Earlier in an experiment on rats, we showed that newly created sulfate-chloride sodium-magnesium drinking mineral waters of Truskavets' spa has a significant modulating effect on the parameters of metabolism and the autonomic nervous, endocrine and immune systems. In this study, we combined data obtained on the same animals, in line with the concepts of neuroendocrine-immune complex and functional-metabolic continuum. Materials and Methods. Experiment was performed on 50 healthy female Wistar rats 230-290 g divided into 4 groups. Animals of the first group remained intact, using tap water from drinking ad libitum. Rats of the second (control) group for 6 days administered a single tap water through the tube at a dose of $1,5 \mathrm{~mL} / 100 \mathrm{~g}$ of body mass. The rats of the main groups received the water "Myroslava" and "Khrystyna". The object of the study were the metabolic, neuro-endocrine and immune parameters. Results. The method of discriminant analysis revealed 31 parameters, according to which all four groups of animals differ from each other. Classification accuracy is $100 \%$. Conclusion. The newly created sulfate-chloride sodiummagnesium drinking mineral waters of Truskavets' spa has both similar and specific effects on the neuroendocrine-immune complex and metabolism at healthy old female rats with weekly use. This provides a basis for preclinical studies.

Keywords: sulfate-chloride sodium-magnesium mineral waters, neuroendocrine-immune complex, metabolism, female rats. 


\section{INRODUCTION}

Earlier in an experiment on rats, we showed that newly created sulfate-chloride sodiummagnesium drinking mineral waters "Myroslava" (5 g/L) and "Khrystyna" (10 g/L) of Truskavets' spa has a significant modulating effects on the parameters of metabolism and the autonomic nervous and endocrine systems [5,6] as well as immunity [1]. In this study, we combined data obtained on the same animals, in line with the concepts of neuroendocrineimmune complex [3,8,12-14] and functional-metabolic continuum [2].

\section{MATERIALS AND METHODS}

Experiment was performed on 50 healthy old female Wistar rats 220-300 g $(\mathrm{M} \pm \mathrm{SD}=262 \pm 23 \mathrm{~g})$ divided into 4 groups. Animals of the first group (10) remained intact, using tap water from drinking ad libitum. Rats of the second (control) group (10) for 6 days administered a single tap water through the tube at a dose of $1,5 \mathrm{~mL} / 100 \mathrm{~g}$ of body mass. The rats of the main groups received the water "Myroslava" (15) and "Khrystyna" (15), prepared from the brine of the $27-K$ well of the Truskavetsian field by appropriate dilutions with fresh water [5]. The object of the study were the metabolic, neuro-endocrine $[5,6]$ and immune [1] parameters

Digital material is statistically processed on a computer using the software package "Statistica 64".

\section{RESULTS AND DISCUSION}

Among the registered parameters, 7 neuroendocrine, 9 metabolic and 15 immune parameters (Tables 1 and 2) were identified by the method of discriminant analysis [7] (forward stepwise program), according to which the intact, control and two main groups of animals differ significantly from each other.

Table 1. Discriminant Function Analysis Summary

Step 31, N of Variables currently in the model: 31; Grouping: 4 groups

Wilks' Lambda: 0,00387; approx. $F_{(93)}=2,83 ; p=0,0001$

\begin{tabular}{|c|c|c|c|c|c|c|c|c|c|}
\hline \multirow[b]{2}{*}{$\begin{array}{l}\text { Variables } \\
\text { currently in the model }\end{array}$} & \multicolumn{4}{|c|}{ Groups (n) } & \multicolumn{5}{|c|}{ Parameters of Wilks' Statistics } \\
\hline & $\begin{array}{l}\text { Khry } \\
\text { styna } \\
\text { (15) }\end{array}$ & $\begin{array}{l}\text { Myro } \\
\text { slava } \\
\text { (15) }\end{array}$ & $\begin{array}{l}\text { Daily } \\
\text { Water } \\
\text { (10) }\end{array}$ & $\begin{array}{l}\text { Intact } \\
\text { rats } \\
(10)\end{array}$ & $\begin{array}{l}\text { Wil } \\
\text { ks' } \\
\Lambda\end{array}$ & $\begin{array}{l}\text { Par- } \\
\text { tial } \\
\Lambda\end{array}$ & $\begin{array}{l}\text { F-re- } \\
\text { move }\end{array}$ & $\begin{array}{l}\text { p- } \\
\text { level }\end{array}$ & $\begin{array}{l}\text { Tole- } \\
\text { rancy }\end{array}$ \\
\hline $\begin{array}{l}\text { Calcium } \\
\text { Plasma, } \\
\mathrm{mM} / \mathrm{L}\end{array}$ & $\begin{array}{l}2,51 \\
0,75 \\
-0,83\end{array}$ & $\begin{array}{l}2,91 \\
0,87 \\
-0,43\end{array}$ & $\begin{array}{l}2,08 \\
0,62 \\
-1,24\end{array}$ & $\begin{array}{l}3,35 \\
1 \\
0\end{array}$ & 0,004 & 0,910 & 0,52 & 0,672 & 0,361 \\
\hline $\begin{array}{l}\text { Superoxide Dismutase } \\
\text { Erythrocytes, } \\
\text { un } / \mathrm{mL}\end{array}$ & $\begin{array}{l}57,7 \\
0,99 \\
-0,03\end{array}$ & $\begin{array}{l}49,9 \\
0,86 \\
-0,75\end{array}$ & $\begin{array}{l}58,2 \\
1,00 \\
+0,02\end{array}$ & $\begin{array}{l}58,0 \\
1 \\
0\end{array}$ & 0,005 & 0,814 & 1,22 & 0,335 & 0,263 \\
\hline $\begin{array}{l}\text { Microbial Count } \\
\text { Neutrophils, } \\
\text { Bacteria/Phagocyte }\end{array}$ & $\begin{array}{l}7,6 \\
0,88 \\
-0,54\end{array}$ & $\begin{array}{l}7,3 \\
0,84 \\
-0,70\end{array}$ & $\begin{array}{l}8,2 \\
0,95 \\
-0,21\end{array}$ & $\begin{array}{l}8,6 \\
1 \\
0\end{array}$ & 0,005 & 0,822 & 1,16 & 0,357 & 0,070 \\
\hline $\begin{array}{l}\text { Sodium } \\
\text { Excretion, } \\
\mu \mathrm{M} / 24 \mathrm{~h} \cdot 100 \mathrm{~g} \text { Body Mass }\end{array}$ & $\begin{array}{l}271 \\
2,01 \\
+1,62\end{array}$ & $\begin{array}{l}167 \\
1,24 \\
+0,39\end{array}$ & $\begin{array}{l}76 \\
0,56 \\
-0,70\end{array}$ & $\begin{array}{l}135 \\
1 \\
0\end{array}$ & 0,005 & 0,782 & 1,49 & 0,255 & 0,057 \\
\hline $\begin{array}{l}\text { Monocytes } \\
\text { Blood, } \\
\%\end{array}$ & $\begin{array}{l}5,07 \\
1,06 \\
+0,09\end{array}$ & $\begin{array}{l}4,87 \\
1,01 \\
+0,02\end{array}$ & $\begin{array}{l}, 20 \\
0,88 \\
-0,20\end{array}$ & $\begin{array}{l}4,80 \\
1 \\
0\end{array}$ & 0,006 & 0,655 & 2,81 & 0,073 & 0,053 \\
\hline Eosinophiles & 4,00 & 3,33 & 3,80 & 4,60 & 0,007 & 0,550 & 4,37 & 0,020 & 0,267 \\
\hline
\end{tabular}




\begin{tabular}{|c|c|c|c|c|c|c|c|c|c|}
\hline $\begin{array}{l}\text { Blood, } \\
\%\end{array}$ & $\begin{array}{l}0,87 \\
-0,20 \\
\end{array}$ & $\begin{array}{l}0,72 \\
-0,42 \\
\end{array}$ & $\begin{array}{l}0,83 \\
-0,27 \\
\end{array}$ & $\begin{array}{l}1 \\
0 \\
\end{array}$ & & & & & \\
\hline $\begin{array}{l}\text { Potassium } \\
\text { Plasma, } \\
\mathrm{mM} / \mathrm{L}\end{array}$ & $\begin{array}{l}3,33 \\
0,79 \\
-1,27\end{array}$ & $\begin{array}{l}3,42 \\
0,81 \\
-1,15\end{array}$ & $\begin{array}{l}3,54 \\
0,84 \\
-0,98\end{array}$ & $\begin{array}{l}4,23 \\
1 \\
0\end{array}$ & 0,006 & 0,647 & 2,91 & 0,067 & 0,344 \\
\hline $\begin{array}{l}(\mathrm{Cap} / \mathrm{Pp})^{0,5} \\
\text { as Parathyroid } \\
\text { Activity }\end{array}$ & $\begin{array}{l}1,75 \\
0,68 \\
-0,70\end{array}$ & $\begin{array}{l}1,91 \\
0,75 \\
-0,56\end{array}$ & $\begin{array}{l}1,58 \\
0,62 \\
-0,84\end{array}$ & $\begin{array}{l}2,56 \\
1 \\
0\end{array}$ & 0,008 & 0,478 & 5,82 & 0,007 & 0,181 \\
\hline $\begin{array}{l}\text { Testosterone } \\
\text { Plasma, } \\
\mathrm{nM} / \mathrm{L}\end{array}$ & $\begin{array}{l}4,50 \\
1,15 \\
+0,53\end{array}$ & $\begin{array}{l}4,97 \\
1,27 \\
+0,98\end{array}$ & $\begin{array}{l}6,04 \\
1,54 \\
+1,97\end{array}$ & $\begin{array}{l}3,93 \\
1 \\
0\end{array}$ & 0,007 & 0,518 & 4,96 & 0,013 & 0,193 \\
\hline $\begin{array}{l}\text { NK Lymphocytes } \\
\text { Blood, } \\
\%\end{array}$ & $\begin{array}{l}16,1 \\
1,03 \\
+0,15\end{array}$ & $\begin{array}{l}16,3 \\
1,04 \\
+0,23\end{array}$ & $\begin{array}{l}14,8 \\
0,95 \\
-0,30\end{array}$ & $\begin{array}{l}15,6 \\
1 \\
0\end{array}$ & 0,006 & 0,698 & 2,30 & 0,116 & 0,043 \\
\hline $\begin{array}{l}\text { Malondialdehyde } \\
\text { Urine, } \\
\mu \mathrm{M} / \mathrm{L}\end{array}$ & $\begin{array}{l}96 \\
1,04 \\
+0,09\end{array}$ & $\begin{array}{l}88 \\
0,95 \\
-0,10\end{array}$ & $\begin{array}{l}75 \\
0,81 \\
-0,40\end{array}$ & $\begin{array}{l}92 \\
1 \\
0\end{array}$ & 0,007 & 0,528 & 4,77 & 0,015 & 0,127 \\
\hline $\begin{array}{l}\text { Leukocytes } \\
\text { Blood, } \\
10^{9} / \mathrm{L}\end{array}$ & $\begin{array}{l}11,76 \\
0,93 \\
-0,15\end{array}$ & $\begin{array}{l}10,51 \\
0,83 \\
-0,36\end{array}$ & $\begin{array}{l}12,55 \\
0,99 \\
-0,02\end{array}$ & $\begin{array}{l}12,68 \\
1 \\
0\end{array}$ & 0,004 & 0,920 & 0,47 & 0,710 & 0,510 \\
\hline $\begin{array}{l}\text { Spleen } \\
\text { Mass Index, } \\
\text { mg/100g Body Mass }\end{array}$ & $\begin{array}{l}312 \\
1,00 \\
0,00\end{array}$ & $\begin{array}{l}268 \\
0,86 \\
-0,44\end{array}$ & $\begin{array}{l}294 \\
0,94 \\
-0,18\end{array}$ & $\begin{array}{l}312 \\
1 \\
0\end{array}$ & 0,004 & 0,902 & 0,58 & 0,635 & 0,365 \\
\hline $\begin{array}{l}\text { Amylase Activity } \\
\text { Urine, } \\
\mathrm{g} / \mathrm{h} \cdot \mathrm{L}\end{array}$ & $\begin{array}{l}204 \\
1,01 \\
+0,02\end{array}$ & $\begin{array}{l}204 \\
1,01 \\
0,04\end{array}$ & $\begin{array}{l}217 \\
1,07 \\
+0,26 \\
\end{array}$ & $\begin{array}{l}202 \\
1 \\
0\end{array}$ & 0,009 & 0,437 & 6,86 & 0,003 & 0,092 \\
\hline $\begin{array}{l}\text { Katalase Activity } \\
\text { Plasma, } \\
\mu \mathrm{M} / \mathrm{h} \cdot \mathrm{L}\end{array}$ & $\begin{array}{l}128 \\
1,24 \\
+0,88\end{array}$ & $\begin{array}{l}122 \\
1,18 \\
+0,67\end{array}$ & $\begin{array}{l}148 \\
1,43 \\
+1,58 \\
\end{array}$ & $\begin{array}{l}103 \\
1 \\
0\end{array}$ & 0,007 & 0,556 & 4,25 & 0,022 & 0,219 \\
\hline $\begin{array}{l}\text { Chloride } \\
\text { Excretion, } \\
\mu \mathrm{M} / 24 \mathrm{~h} \cdot 100 \mathrm{~g} \text { Body Mass }\end{array}$ & $\begin{array}{l}244 \\
1,69 \\
+1,02\end{array}$ & $\begin{array}{l}195 \\
1,35 \\
+0,51\end{array}$ & $\begin{array}{l}107 \\
0,74 \\
-0,38\end{array}$ & $\begin{array}{l}144 \\
1 \\
0\end{array}$ & 0,007 & 0,552 & 4,34 & 0,020 & 0,062 \\
\hline $\begin{array}{l}\text { Triiodothyronine } \\
\text { Plasma, } \\
\text { nM/L }\end{array}$ & $\begin{array}{l}2,38 \\
1,11 \\
+0,42\end{array}$ & $\begin{array}{l}2,31 \\
1,08 \\
+0,30\end{array}$ & $\begin{array}{l}2,11 \\
0,99 \\
-0,05\end{array}$ & $\begin{array}{l}2,14 \\
1 \\
0\end{array}$ & 0,006 & 0,677 & 2,55 & 0,092 & 0,045 \\
\hline $\begin{array}{l}\text { Corticosterone } \\
\text { Plasma, } \\
\mathrm{nM} / \mathrm{L}\end{array}$ & $\begin{array}{l}460 \\
0,96 \\
-0,17\end{array}$ & $\begin{array}{l}365 \\
0,76 \\
-0,92\end{array}$ & $\begin{array}{l}383 \\
0,80 \\
-0,78\end{array}$ & $\begin{array}{l}482 \\
1 \\
0\end{array}$ & 0,006 & 0,684 & 2,46 & 0,100 & 0,332 \\
\hline $\begin{array}{l}\text { Glucose } \\
\text { Plasma, } \\
\mathrm{mM} / \mathrm{L}\end{array}$ & $\begin{array}{l}5,22 \\
1,05 \\
+0,25\end{array}$ & $\begin{array}{l}5,55 \\
1,12 \\
+0,55\end{array}$ & $\begin{array}{l}5,49 \\
1,11 \\
+0,49\end{array}$ & $\begin{array}{l}4,95 \\
1 \\
0\end{array}$ & 0,006 & 0,641 & 2,98 & 0,063 & 0,265 \\
\hline $\begin{array}{l}\text { Phagocytic Index } \\
\text { Monocytes } \\
\%\end{array}$ & $\begin{array}{l}2,89 \\
1,00 \\
-0,01\end{array}$ & $\begin{array}{l}2,83 \\
0,98 \\
-0,10\end{array}$ & $\begin{array}{l}2,75 \\
0,95 \\
-0,21\end{array}$ & $\begin{array}{l}2,90 \\
1 \\
0\end{array}$ & 0,006 & 0,687 & 2,43 & 0,103 & 0,269 \\
\hline $\begin{array}{l}\text { Sodium } \\
\text { Erythrocytes, } \\
\mathrm{mM} / \mathrm{L}\end{array}$ & $\begin{array}{l}24,2 \\
1,10 \\
+0,51\end{array}$ & $\begin{array}{l}21,8 \\
0,99 \\
-0,04\end{array}$ & $\begin{array}{l}22,6 \\
1,03 \\
+0,13\end{array}$ & $\begin{array}{l}22,0 \\
1 \\
0\end{array}$ & 0,006 & 0,600 & 3,56 & 0,038 & 0,116 \\
\hline $\begin{array}{l}\text { Amylase Activity } \\
\text { Plasma, } \\
\mathrm{g} / \mathrm{h} \cdot \mathrm{L}\end{array}$ & $\begin{array}{l}163 \\
1,07 \\
+0,46\end{array}$ & $\begin{array}{l}155 \\
1,02 \\
+0,14\end{array}$ & $\begin{array}{l}154 \\
1,02 \\
+0,10\end{array}$ & $\begin{array}{l}152 \\
1 \\
0\end{array}$ & 0,005 & 0,717 & 2,10 & 0,140 & 0,266 \\
\hline $\begin{array}{l}\text { Macrophages } \\
\text { Spleen, } \\
\%\end{array}$ & $\begin{array}{l}8,1 \\
1,03 \\
+0,15\end{array}$ & $\begin{array}{l}7,9 \\
1,00 \\
+0,02\end{array}$ & $\begin{array}{l}9,1 \\
1,15 \\
+0,75\end{array}$ & $\begin{array}{l}7,9 \\
1 \\
0\end{array}$ & 0,005 & 0,759 & 1,70 & 0,208 & 0,247 \\
\hline $\begin{array}{l}\text { Phagocytic Index } \\
\text { Neutrophils, }\end{array}$ & $\begin{array}{l}69,4 \\
1,00\end{array}$ & $\begin{array}{l}68,9 \\
0,99\end{array}$ & $\begin{array}{l}71,9 \\
1,03\end{array}$ & $\begin{array}{l}69,5 \\
1\end{array}$ & 0,007 & 0,533 & 4,67 & 0,016 & 0,092 \\
\hline
\end{tabular}




\begin{tabular}{|c|c|c|c|c|c|c|c|c|c|}
\hline$\%$ & $-0,03$ & $-0,13$ & $+0,56$ & 0 & & & & & \\
\hline $\begin{array}{l}\text { Reticular } \\
\text { Zone of Adrenal Cortex, } \\
\mu \mathrm{M}\end{array}$ & $\begin{array}{l}42 \\
0,98 \\
-0,12\end{array}$ & $\begin{array}{l}44 \\
1,04 \\
+0,20\end{array}$ & $\begin{array}{l}40 \\
0,95 \\
-0,29\end{array}$ & $\begin{array}{l}43 \\
1 \\
0\end{array}$ & 0,006 & 0,609 & 3,42 & 0,043 & 0,219 \\
\hline $\begin{array}{l}\text { Entropy } \\
\text { Leukocytogram }\end{array}$ & $\begin{array}{l}0,551 \\
0,93 \\
-0,76\end{array}$ & $\begin{array}{l}0,592 \\
0,99 \\
-0,07\end{array}$ & $\begin{array}{l}0,557 \\
0,94 \\
-0,66\end{array}$ & $\begin{array}{l}0,596 \\
1 \\
0\end{array}$ & 0,006 & 0,622 & 3,24 & 0,050 & 0,295 \\
\hline $\begin{array}{l}\text { Plasmocytes } \\
\text { Thymus, } \\
\%\end{array}$ & $\begin{array}{l}2,00 \\
1,11 \\
+0,25\end{array}$ & $\begin{array}{l}2,00 \\
1,11 \\
+0,25\end{array}$ & $\begin{array}{l}2,44 \\
1,36 \\
+0,82\end{array}$ & $\begin{array}{l}1,80 \\
1 \\
0\end{array}$ & 0,006 & 0,690 & 2,40 & 0,106 & 0,300 \\
\hline $\begin{array}{l}\text { Eosinophiles } \\
\text { Spleen, } \\
\%\end{array}$ & $\begin{array}{l}1,14 \\
0,76 \\
-0,33\end{array}$ & $\begin{array}{l}1,73 \\
1,16 \\
+0,22\end{array}$ & $\begin{array}{l}1,40 \\
0,93 \\
-0,09\end{array}$ & $\begin{array}{l}1,50 \\
1 \\
0\end{array}$ & 0,006 & 0,684 & 2,46 & 0,100 & 0,352 \\
\hline $\begin{array}{l}\text { Glomerular Zone } \\
\text { of Adrenal Cortex, } \\
\mu \mathrm{M}\end{array}$ & $\begin{array}{l}185 \\
0,96 \\
-0,18\end{array}$ & $\begin{array}{l}182 \\
0,94 \\
-0,25\end{array}$ & $\begin{array}{l}207 \\
1,07 \\
+0,29\end{array}$ & $\begin{array}{l}193 \\
1 \\
0\end{array}$ & 0,006 & 0,651 & 2,87 & 0,069 & 0,298 \\
\hline $\begin{array}{l}(\mathrm{Ku} / \mathrm{Nau})^{0,5} \\
\text { as Mineralocorticoid } \\
\text { Activity }\end{array}$ & $\begin{array}{l}1,42 \\
0,99 \\
-0,02\end{array}$ & $\begin{array}{l}1,37 \\
0,95 \\
-0,08\end{array}$ & $\begin{array}{l}2,34 \\
1,63 \\
+1,09\end{array}$ & $\begin{array}{l}1,44 \\
1 \\
0\end{array}$ & 0,005 & 0,781 & 1,49 & 0,254 & 0,172 \\
\hline $\begin{array}{l}\text { Magnesium } \\
\text { Urine, } \\
\mathrm{mM} / \mathrm{L}\end{array}$ & $\begin{array}{l}2,89 \\
1,13 \\
+0,18\end{array}$ & $\begin{array}{l}2,49 \\
0,97 \\
-0,04\end{array}$ & $\begin{array}{l}2,34 \\
0,91 \\
-0,12\end{array}$ & $\begin{array}{l}2,56 \\
1 \\
0\end{array}$ & 0,005 & 0,818 & 1,19 & 0,346 & 0,118 \\
\hline
\end{tabular}

Note. In each column, the first line is the average value, the second is the fraction of the norm, and the third is the Z-score.

Table 2. Summary of Stepwise Analysis

\begin{tabular}{|c|c|c|c|c|c|}
\hline $\begin{array}{l}\text { Variables } \\
\text { currently in the model }\end{array}$ & $\begin{array}{l}\text { F to } \\
\text { enter }\end{array}$ & $\begin{array}{l}\text { p- } \\
\text { level }\end{array}$ & $\Lambda$ & $\begin{array}{l}\text { F- } \\
\text { value }\end{array}$ & $\begin{array}{l}\text { p- } \\
\text { level }\end{array}$ \\
\hline Calcium Plasma, $\mathrm{mM} / \mathrm{L}$ & 4,49 & 0,008 & 0,773 & 4,49 & 0,008 \\
\hline Superoxide Dismutase Erythrocytes, un $/ \mathrm{mL}$ & 4,18 & 0,011 & 0,605 & 4,29 & 0,001 \\
\hline Microbial Count Neutrophils, Bac/Phag & 3,38 & 0,027 & 0,492 & 4,03 & $10^{-4}$ \\
\hline Sodium Excretion, $\mu \mathrm{M} / 24 \mathrm{~h} \cdot 100 \mathrm{~g}$ Body Mass & 3,88 & 0,015 & 0,387 & 4,10 & $10^{-4}$ \\
\hline Monocytes Blood, \% & 3,07 & 0,038 & 0,317 & 4,00 & $10^{-5}$ \\
\hline Eosinophiles Blood, \% & 2,49 & 0,074 & 0,268 & 3,83 & $10^{-5}$ \\
\hline Potassium Plasma, $\mathrm{mM} / \mathrm{L}$ & 2,04 & 0,124 & 0,233 & 3,63 & $10^{-5}$ \\
\hline$(\mathrm{Cap} / \mathrm{Pp})^{0,5}$ as Parathyroid Activity & 2,68 & 0,060 & 0,193 & 3,62 & $10^{-5}$ \\
\hline Testosterone Plasma, $\mathrm{nM} / \mathrm{L}$ & 2,07 & 0,121 & 0,166 & 3,51 & $10^{-5}$ \\
\hline NK Lymphocytes Blood, \% & 2,21 & 0,103 & 0,141 & 3,46 & $10^{-6}$ \\
\hline Malondialdehyde Urine, $\mu \mathrm{M} / \mathrm{L}$ & 2,37 & 0,087 & 0,118 & 3,46 & $10^{-6}$ \\
\hline Leukocytes Blood, $10^{9} / \mathrm{L}$ & 1,69 & 0,186 & 0,103 & 3,36 & $10^{-6}$ \\
\hline Spleen Mass Index, mg/100g Body Mass & 1,70 & 0,185 & 0,089 & 3,28 & $10^{-6}$ \\
\hline Amylase Activity Urine, $\mathrm{g} / \mathrm{h} \cdot \mathrm{L}$ & 1,79 & 0,168 & 0,077 & 3,23 & $10^{-6}$ \\
\hline Katalase Activity Plasma, $\mu \mathrm{M} / \mathrm{h} \cdot \mathrm{L}$ & 1,25 & 0,307 & 0,069 & 3,12 & $10^{-5}$ \\
\hline Chloride Excretion, $\mu \mathrm{M} / 24 \mathrm{~h} \bullet 100 \mathrm{~g}$ Body Mass & 1,74 & 0,179 & 0,059 & 3,09 & $10^{-5}$ \\
\hline Triiodothyronine Plasma, nM/L & 1,57 & 0,217 & 0,051 & 3,04 & $10^{-5}$ \\
\hline Corticosterone Plasma, nM/L & 1,55 & 0,224 & 0,044 & 3,00 & $10^{-5}$ \\
\hline Glucose Plasma, $\mathrm{mM} / \mathrm{L}$ & 1,31 & 0,292 & 0,038 & 2,93 & $10^{-5}$ \\
\hline Phagocytic Index Monocytes, \% & 1,38 & 0,270 & 0,033 & 2,89 & $10^{-5}$ \\
\hline Sodium Erythrocytes, $\mathrm{mM} / \mathrm{L}$ & 1,63 & 0,207 & 0,028 & 2,88 & $10^{-5}$ \\
\hline Amylase Activity Plasma, $\mathrm{g} / \mathrm{h} \cdot \mathrm{L}$ & 1,59 & 0,217 & 0,024 & 2,87 & $10^{-5}$ \\
\hline Macrophages Spleen, \% & 1,11 & 0,363 & 0,021 & 2,80 & $10^{-5}$ \\
\hline Phagocytic Index Neutrophils, $\%$ & 1,27 & 0,308 & 0,018 & 2,76 & $10^{-5}$ \\
\hline Reticular Zone of Adrenal Cortex, $\mu \mathrm{M}$ & 1,61 & 0,216 & 0,015 & 2,77 & $10^{-5}$ \\
\hline Entropy Leukocytogram & 1,43 & 0,262 & 0,012 & 2,76 & $10^{-4}$ \\
\hline Plasmocytes Thymus, \% & 1,38 & 0,279 & 0,010 & 2,74 & $10^{-4}$ \\
\hline
\end{tabular}




\begin{tabular}{|l|l|l|l|l|l|}
\hline Eosinophiles Spleen, \% & 1,36 & 0,285 & 0,008 & 2,73 & $10^{-4}$ \\
\hline Glomerular Zone of Adrenal Cortex, $\mu \mathrm{M}$ & 2,76 & 0,072 & 0,006 & 2,92 & $10^{-4}$ \\
\hline$(\mathrm{Ku} / \mathrm{Nau})^{0,5}$ as Mineralocorticoid Activity & 1,09 & 0,381 & 0,005 & 2,87 & $10^{-4}$ \\
\hline Magnesium Urine, $\mathrm{mM} / \mathrm{L}$ & 1,19 & 0,346 & 0,004 & 2,83 & $10^{-4}$ \\
\hline
\end{tabular}

The dividing information contained in 31 variables is condensed in 3 canonical discriminant roots (Tables 3 and 4). The first root contains 53,0\% of discriminative opportunities $\left(\mathrm{r}^{*}=0,950\right.$; Wilks' $\left.\Lambda=0,0039 ; \chi^{2}{ }_{(93)}=175 ; \mathrm{p}<10^{-6}\right)$, the second $28,9 \%\left(\mathrm{r}^{*}=0,914\right.$; Wilks' $\left.\Lambda=0,0397 ; \chi_{(60)}^{2}=100 ; \mathrm{p}=0,0006\right)$, the third $18,1 \% \quad\left(\mathrm{r}^{*}=0,871 ; W i l k s^{\prime} \Lambda=0,2406\right.$; $\left.\chi_{(29)}^{2}=45 ; \mathrm{p}=0,030\right)$.

The calculation of the discriminant root values for each animal as the sum of the products of raw coefficients (Table 3 ) to the individual values of discriminant variables together with the constants enables the visualization of each rat in the information space of the roots (Fig. $1)$.

Table 3. Standardized and Raw Coefficients for Canonical Variables

\begin{tabular}{|c|c|c|c|c|c|c|}
\hline Coefficients & \multicolumn{3}{|c|}{ Standardized } & \multicolumn{3}{|c|}{ Raw } \\
\hline Variables & Root 1 & Root 2 & Root 3 & Root 1 & Root 2 & Root 3 \\
\hline Calcium Plasma, mM/L & 0,485 & $-0,198$ & $-0,058$ & 0,593 & $-0,243$ & $-0,071$ \\
\hline Superoxide Dismutase Erythrocytes, un $/ \mathrm{mL}$ & $-0,012$ & 0,701 & $-0,625$ & $-0,0013$ & 0,079 & $-0,070$ \\
\hline Microbial Count Neutrophils, Bac/Phag & 1,398 & 0,690 & 0,702 & 1,055 & 0,521 & 0,530 \\
\hline Sodium Excretion, $\mu \mathrm{M} / 24 \mathrm{~h} \cdot 100 \mathrm{~g}$ & 1,779 & $-0,952$ & 0,524 & 0,010 & $-0,0055$ & 0,0031 \\
\hline Monocytes Blood, \% & 2,493 & $-0,441$ & $-1,006$ & 1,021 & $-0,180$ & $-0,412$ \\
\hline Eosinophiles Blood, \% & 1,251 & 0,526 & $-0,241$ & 0,612 & 0,257 & $-0,118$ \\
\hline Potassium Plasma, $\mathrm{mM} / \mathrm{L}$ & 1,027 & 0,285 & $-0,079$ & 1,344 & 0,373 & $-0,104$ \\
\hline$(\mathrm{Cap} / \mathrm{Pp})^{0,5}$ as Parathyroid Activity & 1,245 & $-1,222$ & 0,551 & 1,812 & $-1,779$ & 0,802 \\
\hline Testosterone Plasma, $\mathrm{nM} / \mathrm{L}$ & 1,136 & $-0,912$ & $-0,913$ & 0,549 & $-0,441$ & $-0,442$ \\
\hline NK Lymphocytes Blood, \% & $-1,105$ & 1,289 & 2,461 & $-0,505$ & 0,589 & 1,125 \\
\hline Malondialdehyde Urine, $\mu \mathrm{M} / \mathrm{L}$ & 0,765 & 1,886 & 0,528 & 0,023 & 0,058 & 0,016 \\
\hline Leukocytes Blood, $10^{9} / \mathrm{L}$ & 0,114 & 0,377 & $-0,188$ & 0,024 & 0,078 & $-0,039$ \\
\hline Spleen Mass Index, mg/100g Body Mass & 0,181 & 0,436 & 0,327 & 0,0026 & 0,0063 & 0,0047 \\
\hline Amylase Activity Urine, $\mathrm{g} / \mathrm{h} \cdot \mathrm{L}$ & $-0,408$ & $-2,591$ & 0,686 & $-0,010$ & $-0,065$ & 0,017 \\
\hline Katalase Activity Plasma, $\mu \mathrm{M} / \mathrm{h} \cdot \mathrm{L}$ & $-0,670$ & $-1,366$ & $-0,280$ & $-14,41$ & $-29,38$ & $-6,018$ \\
\hline Chloride Excretion, $\mu \mathrm{M} / 24 \mathrm{~h} \cdot 100 \mathrm{~g}$ & $-2,664$ & 1,002 & 0,090 & $-0,018$ & 0,0069 & 0,0006 \\
\hline Triiodothyronine Plasma, $\mathrm{nM} / \mathrm{L}$ & $-2,288$ & 1,660 & 0,354 & $-5,598$ & 4,062 & 0,866 \\
\hline Corticosterone Plasma, nM/L & $-0,402$ & 0,905 & 0,398 & $-0,0024$ & 0,0055 & 0,0024 \\
\hline Glucose Plasma, mM/L & $-1,140$ & $-0,337$ & 0,335 & $-1,375$ & $-0,407$ & 0,405 \\
\hline Phagocytic Index Monocytes, $\%$ & $-0,764$ & 0,094 & $-0,908$ & $-0,874$ & 0,108 & $-1,038$ \\
\hline Sodium Erythrocytes, $\mathrm{mM} / \mathrm{L}$ & $-0,591$ & 1,534 & 1,238 & $-0,123$ & 0,320 & 0,258 \\
\hline Amylase Activity Plasma, $\mathrm{g} / \mathrm{h} \cdot \mathrm{L}$ & $-0,523$ & 0,715 & 0,714 & $-0,015$ & 0,021 & 0,021 \\
\hline Macrophages Spleen, \% & $-0,904$ & 0,143 & $-0,541$ & $-0,497$ & 0,079 & $-0,298$ \\
\hline Phagocytic Index Neutrophils, \% & $-1,157$ & 2,080 & $-0,598$ & $-0,296$ & 0,533 & $-0,153$ \\
\hline Reticular Zone of Adrenal Cortex, $\mu \mathrm{M}$ & $-0,355$ & $-1,414$ & $-0,007$ & $-0,033$ & $-0,132$ & $-0,001$ \\
\hline Entropy Leukocytogram & 0,754 & $-0,931$ & 0,244 & 12,37 & $-15,26$ & 3,994 \\
\hline Plasmocytes Thymus, $\%$ & $-0,769$ & 0,683 & $-0,384$ & $-1,005$ & 0,892 & $-0,503$ \\
\hline Eosinophiles Spleen, \% & 0,112 & $-0,958$ & 0,397 & 0,131 & $-1,121$ & 0,465 \\
\hline Glomerular Zone of Adrenal Cortex, $\mu \mathrm{M}$ & 0,273 & 1,032 & $-0,530$ & 0,008 & 0,029 & $-0,015$ \\
\hline$(\mathrm{Ku} / \mathrm{Nau})^{0,5}$ as Mineralocorticoid Activity & 1,076 & 0,519 & $-0,008$ & 1,128 & 0,544 & $-0,008$ \\
\hline Magnesium Urine, $\mathrm{mM} / \mathrm{L}$ & 1,208 & 0,111 & $-0,534$ & 0,717 & 0,066 & $-0,317$ \\
\hline & & & onstants & 26,74 & $-54,47$ & $-16,66$ \\
\hline & & & envalues & 9,26 & 5,06 & 3,16 \\
\hline & Cun & lative $\mathbf{P}$ & portions & 0,530 & 0,819 & 1 \\
\hline
\end{tabular}


Table 4. Factor Structure Matrix (Correlations Variables-Canonical Roots) and Means of Roots and Variables Z-scores

\begin{tabular}{|c|c|c|c|c|c|c|c|}
\hline \multirow[b]{2}{*}{ Root $1(53,0 \%)$} & \multicolumn{3}{|c|}{$\begin{array}{l}\text { Correlations Variables- } \\
\text { Roots }\end{array}$} & \multirow{2}{*}{$\begin{array}{l}\begin{array}{l}\text { Khry } \\
\text { styna }\end{array} \\
-1,96 \\
\end{array}$} & \multirow{2}{*}{$\begin{array}{l}\text { Myro } \\
\text { slava }\end{array}$} & \multirow{2}{*}{$\begin{array}{l}\begin{array}{l}\text { Daily } \\
\text { Water }\end{array} \\
-0,41 \\
\end{array}$} & \multirow{2}{*}{$\begin{array}{l}\begin{array}{l}\text { Intact } \\
\text { rats }\end{array} \\
+5,73 \\
\end{array}$} \\
\hline & R1 & R2 & R3 & & & & \\
\hline$(\mathrm{Cap} / \mathrm{Pp})^{0,5}$ as Parathyroid Act & 0,148 & $-0,039$ & 0,127 & $-0,70$ & $-0,56$ & $-0,84$ & $\mathbf{0}$ \\
\hline Calcium Plasma & 0,112 & $-0,084$ & 0,212 & $-0,83$ & $-0,43$ & $-1,24$ & $\mathbf{0}$ \\
\hline Potassium Plasma & 0,149 & $-0,011$ & $-0,003$ & $-1,27$ & $-1,15$ & $-0,98$ & $\mathbf{0}$ \\
\hline Microbial Count Neutrophils & 0,118 & 0,040 & $-0,105$ & $-0,54$ & $-0,70$ & $-0,21$ & $\mathbf{0}$ \\
\hline Eosinophils Blood & 0,061 & 0,057 & 0,006 & $-0,20$ & $-0,42$ & $-0,27$ & $\mathbf{0}$ \\
\hline Glucose Plasma & $-0,070$ & $-0,074$ & $-0,058$ & $+0,25$ & $+0,55$ & $+0,49$ & 0 \\
\hline Katalase Activity Plasma & $-0,068$ & 0,024 & $-0,120$ & $+0,88$ & $+0,67$ & $+1,58$ & 0 \\
\hline Amylase Activity Plasma & $-0,029$ & 0,038 & 0,027 & $+0,46$ & $+0,14$ & $+0,10$ & $\mathbf{0}$ \\
\hline Root $2(28,9 \%)$ & R1 & $\mathbf{R 2}$ & R3 & $+2,64$ & $-2,92$ & $+0,26$ & $+0,15$ \\
\hline Corticosterone Plasma & 0,054 & 0,104 & 0,068 & $-0,17$ & $-0,92$ & $-0,78$ & 0 \\
\hline SOD Erythrocytes & 0,054 & 0,166 & $-0,082$ & $-0,03$ & $-0,75$ & $+0,02$ & 0 \\
\hline Sodium Erythrocytes & $-0,030$ & 0,087 & 0,021 & $+0,51$ & $-0,04$ & $+0,13$ & 0 \\
\hline Spleen Mass Index & 0,041 & 0,110 & 0,006 & 0,00 & $-0,44$ & $-0,18$ & 0 \\
\hline Leukocytes Blood & 0,042 & 0,044 & $-0,056$ & $-0,15$ & $-0,36$ & $-0,02$ & 0 \\
\hline Eosinophils Spleen & 0,015 & $-0,126$ & 0,002 & $-0,33$ & $+0,22$ & $-0,09$ & 0 \\
\hline Entropy Leukocytogram & 0,060 & $-0,114$ & 0,061 & $-0,76$ & $-0,07$ & $-0,66$ & 0 \\
\hline Root $3(18,1 \%)$ & R1 & R2 & R3 & $+1,06$ & $+0,78$ & $-3,39$ & $+0,63$ \\
\hline Testosterone Plasma & $-0,059$ & $-0,033$ & $-0,165$ & $+0,53$ & $+0,98$ & $+1,97$ & 0 \\
\hline$(\mathrm{Ku} / \mathrm{Nau})^{0,5}$ as MC Activity & $-0,004$ & 0,021 & $-0,228$ & $-0,02$ & $-0,08$ & $+1,09$ & 0 \\
\hline Glomerular ZAC & 0,028 & 0,025 & $-0,136$ & $-0,18$ & $-0,25$ & $+0,29$ & 0 \\
\hline Phagocytic Index Neutrophils & 0,007 & 0,021 & $-0,164$ & $-0,03$ & $-0,13$ & $+0,56$ & 0 \\
\hline Plasmocytes Thymus & $-0,034$ & $-0,011$ & $-0,144$ & $+0,25$ & $+0,25$ & $+0,82$ & 0 \\
\hline Macrophages Spleen & $-0,024$ & 0,045 & $-0,127$ & $+0,15$ & $+0,02$ & $+0,75$ & 0 \\
\hline Amylase Activity Urine & $-0,008$ & $-0,000$ & $-0,068$ & $+0,02$ & $+0,04$ & $+0,26$ & 0 \\
\hline Triiodothyronine Plasma & $-0,063$ & 0,021 & 0,117 & $+0,42$ & $+0,30$ & $-0,05$ & 0 \\
\hline Reticular ZAC & $-0,000$ & $-0,045$ & 0,052 & $-0,12$ & $+0,20$ & $-0,29$ & 0 \\
\hline Sodium Excretion & $-0,062$ & 0,095 & 0,183 & $+1,62$ & $+0,39$ & $-0,70$ & 0 \\
\hline Chloride Excretion & $-0,064$ & 0,049 & 0,163 & $+1,02$ & $+0,51$ & $-0,38$ & 0 \\
\hline Malondialdehyde Urine & 0,004 & 0,037 & 0,115 & $+0,09$ & $-0,10$ & $-0,40$ & 0 \\
\hline Magnesium Urine & $-0,010$ & 0,040 & 0,048 & $+0,18$ & $-0,04$ & $-0,12$ & 0 \\
\hline NK Lymphocytes Blood & $-0,036$ & $-0,002$ & 0,151 & $+0,15$ & $+0,23$ & $-0,30$ & 0 \\
\hline Monocytes Blood & $-0,014$ & 0,029 & 0,084 & $+0,09$ & $+0,02$ & $-0,20$ & 0 \\
\hline Phagocytic Index Monocytes & 0,011 & $-0,001$ & 0,026 & $-0,01$ & $-0,10$ & $-0,21$ & 0 \\
\hline
\end{tabular}



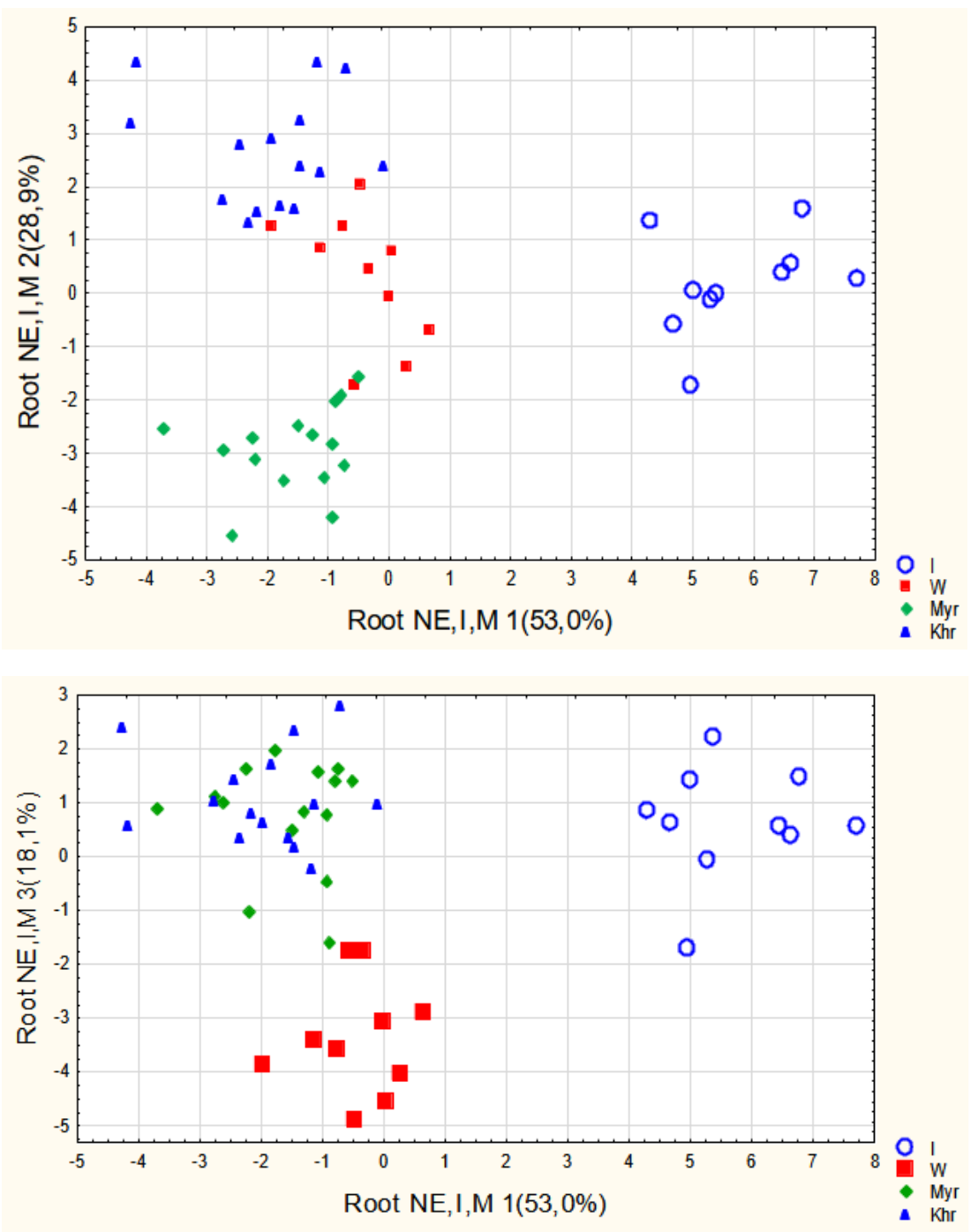

Fig. 1. Individual values of the first and second (above) and the first and third (below) roots of the endocrine and metabolic parameters in intact rats (o) and loaded with Daily water (W) and mineral waters "Myroslava" (Myr) and "Khrystyna" (Khr)

Pseudo-staining visualizes a combination of hormonal, immune, and metabolic parameters in the structure of each root (Table 4), consistent with previously identified neuroendocrine-immune and neuroendocrine-metabolic linkages [11,18-22].

As you can see (Fig. 1 above), along the axis of the first root of the rat, both control and both main groups, significantly distant from intact animals, while their projections on the axis are closely mixed.

This disposition reflects a decrease in parathyroid activity and plasma calcium and potassium levels, as well as eosinophils in the blood and the intensity of bacterial phagocytosis by neutrophils on the one hand, while increased plasma glucose levels and catalase and amylase activity on the other. The described changes are nonspecific and are caused, apparently, by adversarial stress [15,23].

Instead, the groups subjected to water loading are quite clearly delineated along the axis of the second root. The lowest position of "Myroslava" loaded rats showed the maximum decrease in plasma corticosterone, sodium and SOD in erythrocytes, leukocytes in blood and spleen mass in combination with the maximum content in the splenocytogram of eosinophils and maximum entropy of leukocytogram. At the opposite pole of the axis are animals loaded with "Khrystyna" water, and the rats of the control group occupy an intermediate position. 
Obviously, this illustrates the specificity of the modulating effects of mineral waters with different mineralization [4].

Additional delimitation of rats of the control group occurs along the axis of the third root. Their lowest localization reflects elevated or maximal for sampling testosterone levels, mineralocorticoid activity, adrenal glomerular thickness, amylasuria, phagocytic index of blood neutrophils, as well as the content of plasma cells in the thymus and macrophages in the spleen. In contrast, this cluster is characterized by low or minimal sampling levels of triiodothyronine, adrenal reticular thickness, urinary excretion of sodium and chloride, urinary concentrations of magnesium and malonic dialdehyde, as well as phagocytic index of blood monocytes and the content of monocytes and natural killers.

Both mineral waters equally prevent changes in these parameters, which is a manifestation of their non-specific stress limiting effect.

In general, in the information field of the three roots, all four groups of animals are quite different from each other, as documented by the distances of Mahalanobis (Table 5).

Table 5. Squared Mahalanobis Distances between groups (over diagonal), F-values $(\mathrm{df}=\mathbf{3 1})$ and $\mathrm{p}$-levels (under diagonal)

\begin{tabular}{|l|l|l|l|l|}
\hline Groups & $\begin{array}{l}\text { I } \\
(10)\end{array}$ & $\begin{array}{l}\text { DW } \\
(10)\end{array}$ & $\begin{array}{l}\text { Myr } \\
(15)\end{array}$ & $\begin{array}{l}\text { Khr } \\
(15)\end{array}$ \\
\hline Intact rats (I) & $\mathbf{0 , 0}$ & 54 & 63 & 65 \\
\hline $\begin{array}{l}\text { Daily Water } \\
\text { (DW) }\end{array}$ & $\mathbf{3 , 0 3}$ & $\mathbf{0 , 0}$ & 29 & 28 \\
\hline $\begin{array}{l}\text { Water "Myroslava" } \\
\text { (Myr) }\end{array}$ & $\mathbf{4 , 2 5}$ & $\mathbf{1 , 9 5}$ & $\mathbf{0 , 0}$ & 31 \\
\hline $\begin{array}{l}\text { Water "Khrystyna" } \\
\text { (Khr) }\end{array}$ & $\mathbf{4 , 4 1}$ & $\mathbf{1 , 8 8}$ & $\mathbf{2 , 6 1}$ & $\mathbf{0 , 0}$ \\
\hline
\end{tabular}

The application of the classifying functions (Table 6) enables the retrospective identification of all rats without mistake (Table 7).

Table 6. Coefficients and Constants for Classification Functions

\begin{tabular}{|c|c|c|c|c|}
\hline Variables currently in the model & $\begin{array}{l}\text { Intact } \\
\text { rats }\end{array}$ & $\begin{array}{l}\text { Daily } \\
\text { Water }\end{array}$ & $\begin{array}{l}\text { Myro- } \\
\text { slava }\end{array}$ & $\begin{array}{l}\text { Khrys- } \\
\text { tyna }\end{array}$ \\
\hline Calcium Plasma, mM/L & $-29,94$ & $-33,33$ & $-33,55$ & $-35,14$ \\
\hline Superoxide Dismutase Erythrocytes, un/mL & 3,424 & 3,723 & 3,182 & 3,599 \\
\hline Microbial Count Neutrophils, Bac/Phag & 62,39 & 53,83 & 53,14 & 55,80 \\
\hline Sodium Excretion, $\mu \mathrm{M} / 24 \mathrm{~h} \bullet 100 \mathrm{~g}$ BMI & $-0,818$ & $-0,895$ & $-0,877$ & $-0,910$ \\
\hline Monocytes Blood, \% & $-71,75$ & $-76,39$ & $-78,74$ & $-80,23$ \\
\hline Eosinophils Blood, \% & 2,272 & $-0,987$ & $-3,021$ & $-1,847$ \\
\hline Potassium Plasma, mM/L & 17,16 & 9,361 & 6,150 & 7,709 \\
\hline$(\mathrm{Cap} / \mathrm{Pp})^{0,5}$ as Parathyroid Activity & $-124,1$ & $-138,6$ & $-131,8$ & $-142,1$ \\
\hline Testosterone Plasma, $\mathbf{n M} / \mathrm{L}$ & $-46,69$ & $-48,34$ & $-49,44$ & $-52,21$ \\
\hline NK Lymphocytes Blood, \% & 123,9 & 122,5 & 125,9 & 129,7 \\
\hline Malondialdehyde Urine, $\mu \mathrm{M} / \mathrm{L}$ & 2,913 & 2,710 & 2,567 & 2,883 \\
\hline Leukocytes Blood, $10^{9} / \mathrm{L}$ & 7,374 & 7,395 & 6,955 & 7,370 \\
\hline Spleen Mass Index, mg/100g Body Mass & 0,582 & 0,548 & 0,544 & 0,579 \\
\hline Amylase Activity Urine, $\mathrm{g} / \mathrm{h} \cdot \mathrm{L}$ & $-3,227$ & $-3,240$ & $-2,952$ & $-3,302$ \\
\hline Katalase Activity Plasma, $\mu M / h \cdot L$ & -1238 & -1129 & -1043 & -1203 \\
\hline Chloride Excretion, $\mu \mathrm{M} / 24 \mathrm{~h} \bullet 100 \mathrm{~g}$ BM & 1,260 & 1,370 & 1,372 & 1,417 \\
\hline Triiodothyronine Plasma, $\mathrm{nM} / \mathrm{L}$ & 578,3 & 609,7 & 607,0 & 631,9 \\
\hline Corticosterone Plasma, nM/L & 0,494 & 0,500 & 0,495 & 0,527 \\
\hline Glucose Plasma, $\mathrm{mM} / \mathrm{L}$ & 50,91 & 57,69 & 62,29 & 60,65 \\
\hline Phagocytic Index Monocytes, \% & 18,45 & 28,00 & 24,36 & 24,98 \\
\hline
\end{tabular}




\begin{tabular}{|c|c|c|c|c|}
\hline Sodium Erythrocytes, mM/L & 31,46 & 31,22 & 31,42 & 33,32 \\
\hline Amylase Activity Plasma, g/h॰L & 2,534 & 2,546 & 2,584 & 2,710 \\
\hline Macrophages Spleen, \% & 12,83 & 17,09 & 16,19 & 16,72 \\
\hline Phagocytic Index Neutrophils, \% & 52,39 & 54,89 & 52,91 & 55,93 \\
\hline Reticular Zone of Adrenal Cortex, $\mu \mathrm{M}$ & $-6,299$ & $-6,106$ & $-5,649$ & $-6,372$ \\
\hline Entropy Leukocytogram & $-674,7$ & $-768,5$ & $-717,9$ & $-806,0$ \\
\hline Plasmocytes Thymus, \% & 113,4 & 121,7 & 118,0 & 123,1 \\
\hline Eosinophiles Spleen, \% & $-18,41$ & $-21,20$ & $-15,86$ & $-22,00$ \\
\hline Glomerular Zone of Adrenal Cortex, $\mu \mathrm{M}$ & 1,349 & 1,365 & 1,203 & 1,356 \\
\hline$(\mathrm{Ku} / \mathrm{Nau})^{0,5}$ as Mineralocorticoid Activity & $-4,639$ & $-11,47$ & $-14,57$ & $-11,96$ \\
\hline Magnesium Urine, mM/L & $-39,64$ & $-42,77$ & $-45,15$ & $-45,13$ \\
\hline Constants & -3953 & -4046 & -3974 & -4291 \\
\hline
\end{tabular}

Table 11. Classification Matrix

Rows: Observed classifications; Columns: Predicted classifications

\begin{tabular}{|c|c|c|c|c|c|}
\hline \multirow[b]{2}{*}{ Groups } & \multirow{2}{*}{$\begin{array}{l}\text { Percent } \\
\text { correct }\end{array}$} & $\mathrm{I}$ & DW & Myr & Khr \\
\hline & & $\mathrm{p}=, 20$ & $\mathrm{p}=, 20$ & $\mathrm{p}=, 30$ & $\mathrm{p}=, 30$ \\
\hline Intact rats $(\mathrm{I})$ & 100 & 10 & 0 & 0 & 0 \\
\hline Daily Water (DW) & 100 & 0 & 10 & 0 & 0 \\
\hline Water "Myroslava" (Myr) & 100 & 0 & 0 & 15 & 0 \\
\hline Water "Khrystyna" (Khr) & 100 & 0 & 0 & 0 & 15 \\
\hline $\begin{array}{r}\text { Total } \\
\end{array}$ & 100 & 10 & 10 & 15 & 15 \\
\hline
\end{tabular}

Another approach to identifying the specificity of the effects is to create patterns of Zscores parameters, both included in the discriminant model and extramodel, but carrying recognizable information. Calculating the algebraic difference between Z-scores parameters in control and experimental groups allows us to estimate the partial effects of mineral waters (Fig. 2).

The first pattern shows how both mineral waters equally prevent the stress-induced increase in thickness of the glomerular zone of the adrenal cortex and mineralocorticoid activity, glycemia and amylasuria, thymus mass and content in the thymocytogram of endothelial cells, in the splenocytogram macrophages as well as the phagocytic index of blood neutrophils.

Significantly higher stress-induced four parameters (testosterone, plasma catalase, thymocytogram plasma cells and immunocytogram entropy) under the influence of mineral waters are reduced to the upper zone of normal.

On the other hand (third pattern), they prevent a stress-induced decrease in thickness of the reticular zone of the adrenal cortex, triiodethyroninemia, parathyroid activity, calciumemia, urinary excretion of sodium and chloride, urinary concentration of malonic dialdehyde, as well as blood monocytes count, the activity and intensity of bacterial phagocytosis by monocytes.

The following three patterns reflect the differences in the effects of mineral waters. "Myroslava" water deepens chronic stress-induced decrease in corticosterone, SOD, lymphoblast of thymocytogram content, spleen mass and plasma cell of splenocytogram content, blood content of leukocytes in general and eosinophils in particular as well as the intensity of phagocytosis of bacteria by neutrophils and the transformation of $\mathrm{T}$ lymphocytes 
into blasts. On the other hand, "Khrystyna" water does not affect this constellation of parameters in general.

The next pattern demonstrates that stress-insensitive parameters (amylasemia, natrihistia, magnesiumuria, lymphoblast and reticulocyte content in splenocytogram, $\mathrm{T}$ cytolytic lymphocytes content in immunocytogram, and neutrophil killing index) increase under the influence of "Khrystyna" water while "Myroslava" water is inefficient for these parameters

In contrast, "Myroslava" water, unlike "Khrystyna" water, initiates increase in the entropy of leukocytogram and thymocytogram, level in thymocytogram of epitheliocytes, macrophages and reticulocytes, as well as eosinophils in the splenocytogram, NK lymphocytes in the blood.

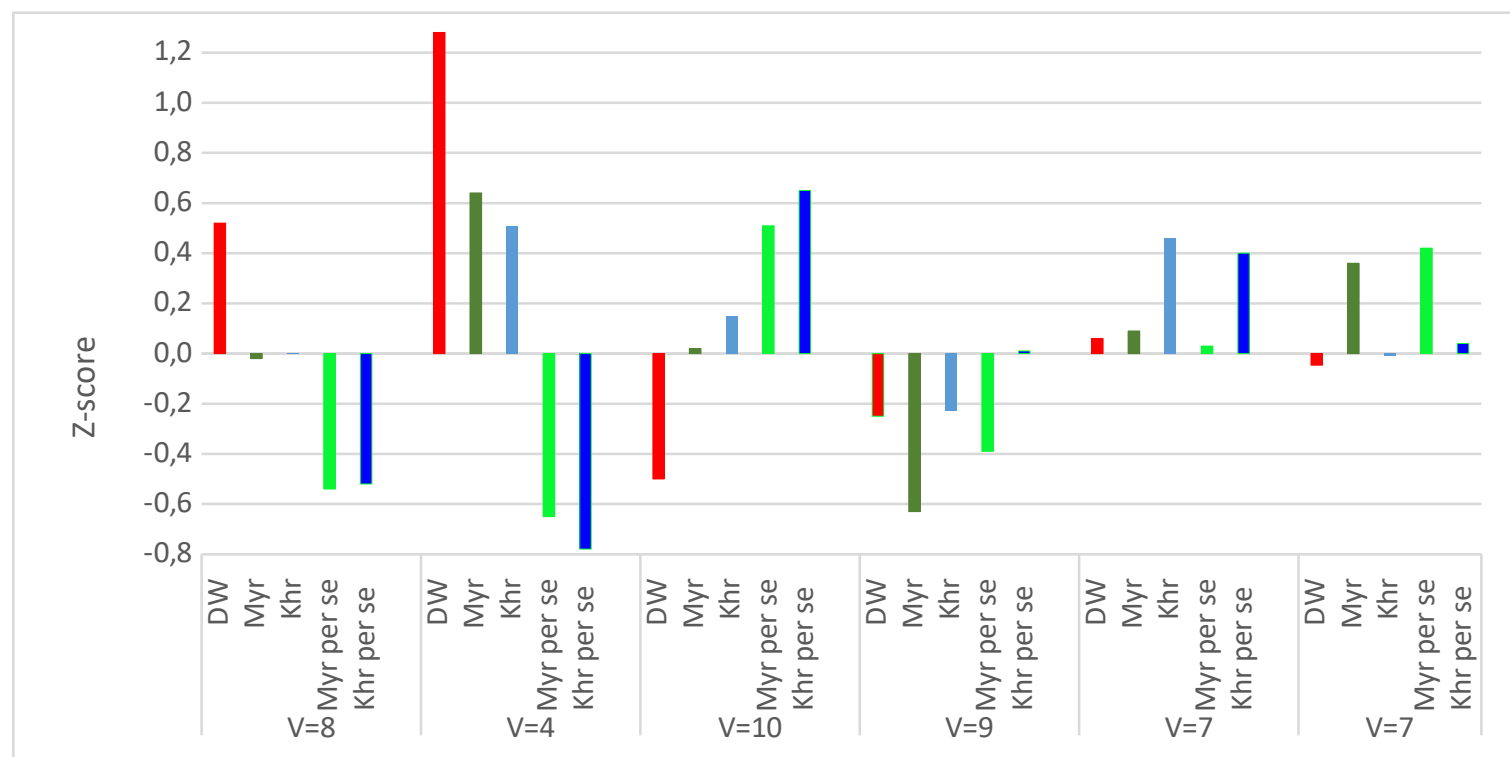

Fig. 2. Patterns ( $V$ - number of variables) of effects of daily water and mineral waters and simulated partial effects of mineral waters

\section{CONCLUSION}

The newly created sulfate-chloride sodium-magnesium drinking mineral waters of Truskavets' spa has both similar and specific effects on the neuroendocrine-immune complex and metabolism at healthy old female rats with weekly use. This provides a basis for preclinical studies.

Based on preliminary data $[9,10,16]$, it is possible to predict the modulating effect of the studied mineral waters on the parameters of the electroencephalogram in humans.

\section{CONFORMITY TO ETHICAL STANDARDS}

Experiments on animals have been carried out in accordance with the provisions of the Helsinki Declaration of 1975, revised and supplemented in 2002 by the Directives of the National Committees for Ethics in Scientific Research.

The conduct of experiments was approved by the Ethics Committee of the Horbachevskyi Ternopil' National Medical University. The modern rules for the maintenance and use of laboratory animals complying with the principles of the European Convention for the Protection of Vertebrate Animals used for scientific experiments and needs are observed (Strasbourg, 1985). 


\section{REFERENCES}

1. Badiuk NS, Popovych DV, Hrytsak MV, Ruzhylo SV, Zakalyak NR, Kovalchuk GY, Mel'nyk OI, Zukow X. Similar and specific immunotropic effects of sulfate-chloride sodium-magnesium mineral waters "Myroslava" and "Khrystyna" of Truskavets' spa in healthy female rats. Journal of Education, Health and Sport. 2021; 11(11): 314-353.

2. Gozhenko AI. Functional-metabolic continuum [in Russian]. J of NAMS of Ukraine. 2016; 22 (1): $3-8$

3. Gozhenko AI, Korda MM, Popadynets' OO, Popovych IL. Entropy, Harmony, Synchronization, Harmony and Their Neuro-Endocrine-Immune Correlates [in Ukrainian]. Odesa. Feniks; 2021: 232.

4. Gozhenko OA, Zavidnyuk YV, Korda MM, Mysula IR, Klishch IM, Zukow W, Popovych IL. Features of neuro-endocrine and immune reactions to various water-salt loads in female rats. Journal of Education, Health and Sport. 2018; 8(9): 11-31.

5. Hrytsak MV, Popovych DV, Badiuk NS, Hrytsan II, Zukow W. Similar neuroendocrine and metabolic effects of sulfate-chloride sodium-magnesium mineral waters "Myroslava" and "Khrystyna" of Truskavets' spa in healthy female rats. Journal of Education, Health and Sport. 2021; 11(6): 320334.

6. Hrytsak MV, Popovych DV, Badiuk NS, Hrytsan II, Zukow W. Peculiaritias of neuroendocrine and metabolic effects of sulfate-chloride sodium-magnesium mineral waters "Myroslava" and "Khrystyna" of Truskavets' spa in healthy female rats. Journal of Education, Health and Sport. 2021; 11(9): 862-875.

7. Klecka WR. Discriminant Analysis [trans. from English in Russian] (Seventh Printing, 1986). In: Factor, Discriminant and Cluster Analysis. Moskva. Finansy i Statistika; 1989: 78-138.

8. Kozyavkina OV, Kozyavkina NV, Gozhenko OA, Gozhenko AI, Barylyak LG., Popovych IL. Bioactive Water Naftussya and Neuro-Endocrine-Immune Complex [in Ukrainian]. Kyiv: UNESCOSOCIO; 2015: 349.

9. Kul'chyns'kyi AB, Kyjenko VM, Zukow W, Popovych IL. Causal neuro-immune relationships at patients with chronic pyelonephritis and cholecystitis. Correlations between parameters EEG, HRV and white blood cell count. Open Medicine. 2017; 12(1): 201-213.

10. Kul'chyns'kyi AB, Zukow W, Korolyshyn TA, Popovych IL. Interrelations between changes in parameters of HRV, EEG and humoral immunity at patients with chronic pyelonephritis and cholecystitis. Journal of Education, Health and Sport. 2017; 7(9): 439-459.

11. Mel'nyk OI, Zukow W, Hrytsak MV, Popovych DV, Zavidnyuk YV, Bilas VR, Popovych IL. Canonical analysis of neuroendocrine-metabolic and neuroendocrine-immune relationships at female rats. Journal of Education, Health and Sport. 2021; 11(5): 356-369.

12. Polovynko IS, Zayats LM, Zukow W, Popovych IL. Neuro-endocrine-immune relationships by chronic stress at male rats. Journal of Health Sciences. 2013; 3(12): 365-374.

13. Popovych IL. Functional interactions between neuroendocrine-immune complex in males rats [in Ukrainian]. Achievements of Clinical and Experimental Medicine. 2008; 2(9): 80-87.

14. Popovych IL. The concept of neuroendocrine-immune complex (Review) [in Russian]. Medical Hydrology and Rehabilitation. 2009; 7(3): 9-18.

15. Popovych IL, Gozhenko AI, Zukow W, Polovynko IS. Variety of Immune Responses to Chronic Stress and their Neuro-Endocrine Accompaniment. Scholars' Press. Riga; 2020: 172.

16. Popovych IL, Kul'chyns'kyi AB, Korolyshyn TA, Zukow W. Interrelations between changes in parameters of HRV, EEG and cellular immunity at patients with chronic pyelonephritis and cholecystitis. Journal of Education, Health and Sport. 2017; 7(10): 11-23.

17. Popovych IL, Vis'tak HI, Gumega MD, Ruzhylo SV. Vegetotropic Effects of Bioactive Water Naftussya and their Endocrine-Immune, Metabolic and Hemodynamic Accompaniments [in Ukrainian]. Kyiv: UNESCO-SOCIO; 2014: 163.

18. Sternberg EM. Neural regulation of innate immunity: a coordinated nonspecific host response to pathogens. Nat Rev Immunol. 2006; 6(4): 318-328.

19. Thayer JF, Sternberg EM. Neural aspects of immunomodulation: Focus on the vagus nerve. Brain Behav Immun. 2010; 24(8): 1223-1228.

20. Tracey KJ. Reflex control of immunity. Nat Rev Immunol. 2009; 9(6): 418-428. 
21. Uchakin PN, Uchakina ON, Tobin BV, Ershov FI. Neuroendocrine immunomodulation [in Russian]. Vestnik Ross AMN. 2007; 9: 26-32.

22. Zajats LM, Polovynko IS, Zukow W. Features neuro-endocrine support diversity of immune responses to chronic stress in male rats. Journal of Education, Health and Sport. 2017; 7(3): 97-105.

23. Zavidnyuk YV, Mysula IR, Klishch IM, Zukow W, Popovych IL, Korda MM. General nonspecific metabolic, neuroendocrine and immune reactions to various water-salt loads in female rats. Journal of Education, Health and Sport. 2018; 8(3): 513-524. 